ClinicAl VISTAS

\title{
Intermittent forearm cyanosis
}

A 42-year-old woman was admitted to the intensive care unit because her right forearm was cyanotic. The patient had received a diagnosis of primary adult respiratory distress syndrome and had been intubated for 2 weeks. A right central venous catheter had been in place for II days, and it had been removed I day before she was readmitted to the intensive care unit.

A physical examination performed while the patient was in a sitting position revealed that her right forearm was tense and swollen, with a blue-purple hue (Figure I). The superficial veins on the dorsal side of the patient's hand were dilated, and they did not empty when it was elevated above her heart. This occurred only when the patient was in a sitting position, and it quickly resolved when she lay down (Figure I).

We used duplex imaging to exclude an acute axillosubclavian vein thrombosis. Results of this test showed normal flow enhancement during deep inspiration when the patient was in a lying position and showed that venous flow was almost flat when the patient was in a sitting position (Figure 2). This result was

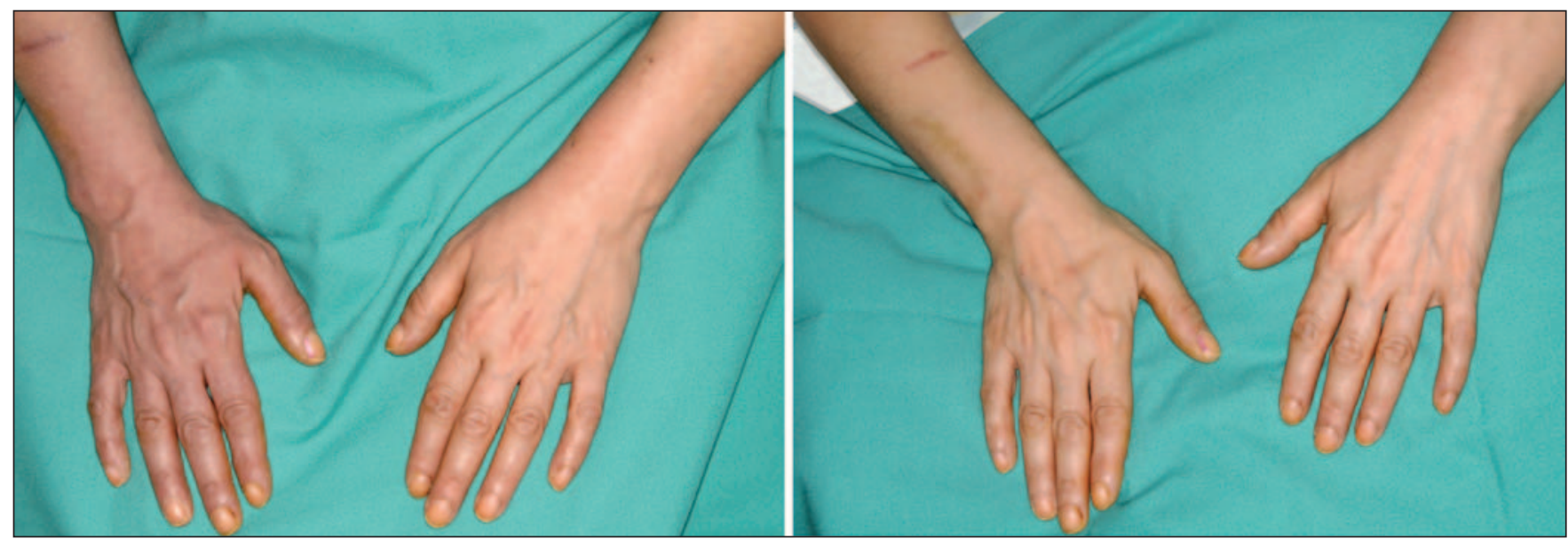

Figure 1: Patient's forearms while in a sitting position (left) and a few seconds after lying down (right).
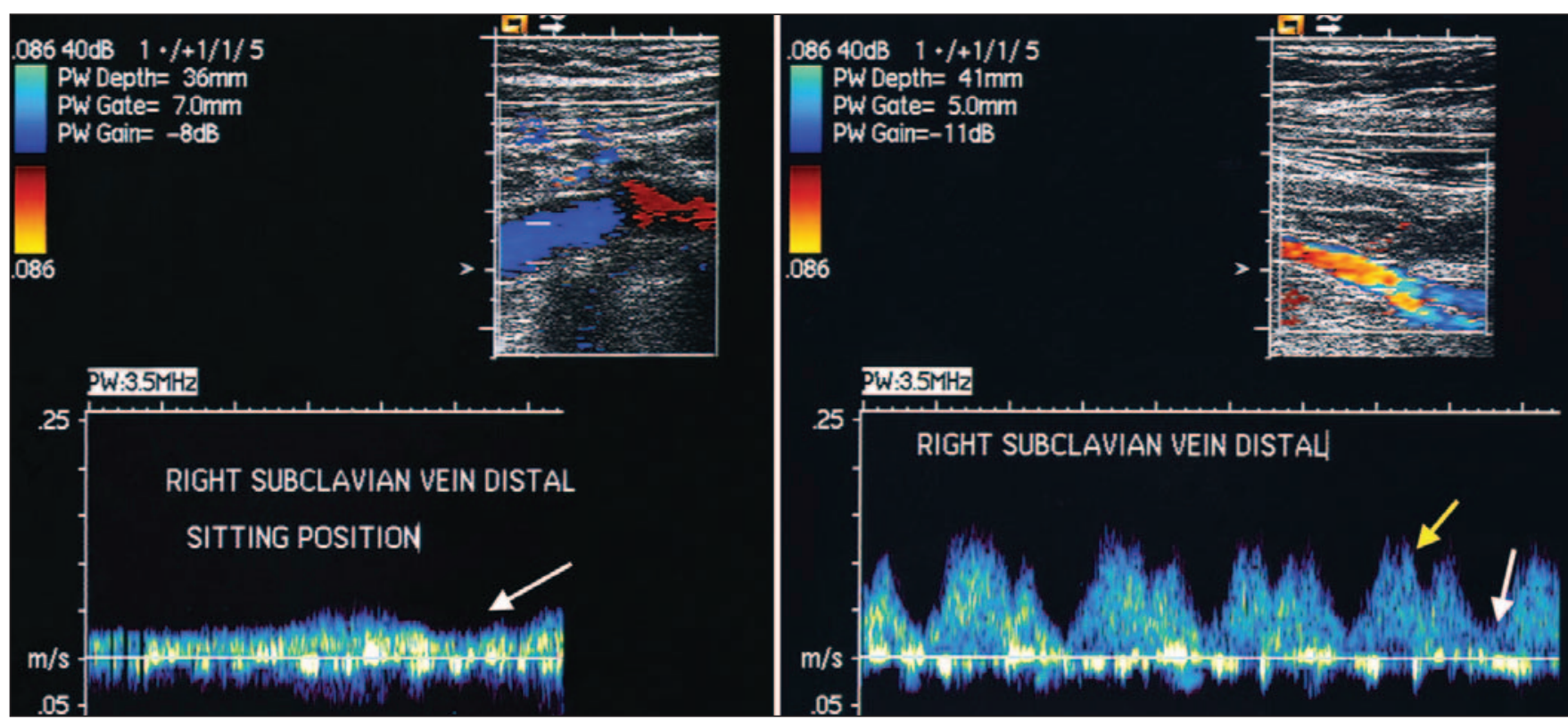

Figure 2: Duplex imaging of the patient's right subclavian vein while in a sitting (left) and lying position (right). Normal flow in the subclavian vein is observed when the patient is in a lying position, and the normal flow enhancement during deep inspiration is lost when the patient is sitting up and venous flow is flat and nearly obliterated. The arrows indicate cardiac and respiratory modulation of the venous flow (white arrow, inspiration; yellow arrow, expiration). The colour of the flow indicates whether blood is flowing toward (blue) or away from (red) the transducer. 


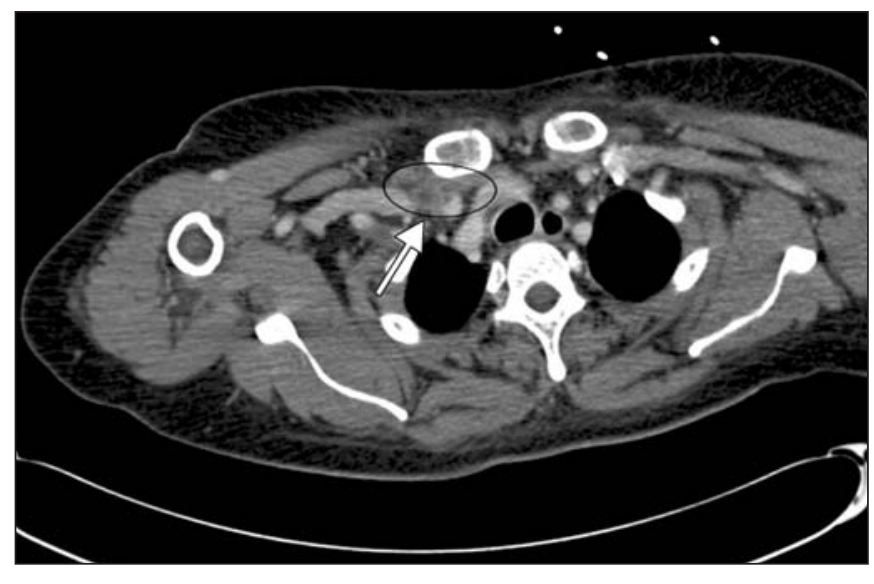

Figure 3: Computed tomography angiogram showing a hematoma at the puncture site under the patient's clavicle (arrow).

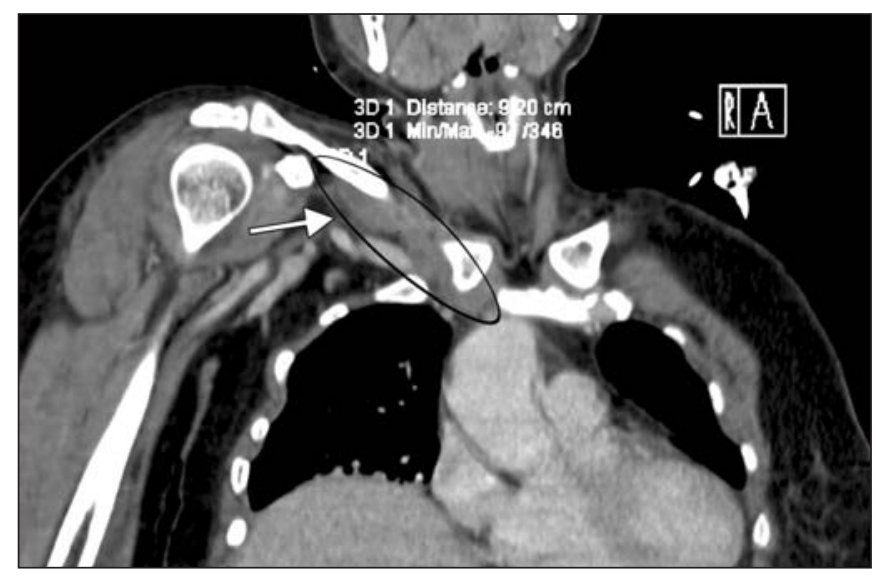

Figure 4: Computed tomography angiogram (right-anterior view) showing the length of the hematoma and its proximity to the clavicle and subclavian vein. reproducible and confirmed the dynamic nature of the venous inflow obstruction. It also explained the positional nature of the patient's symptoms. A computed tomography angiogram revealed a $9 \times 3 \times 2 \mathrm{~cm}$ hematoma involving the subclavian muscle at the catheter puncture site, precisely under the patient's clavicle (Figure 3, Figure 4). The hematoma caused vascular compression when the patient was in an upright position because it was precisely beneath the clavicle and adjacent to the subclavian vein. The patient also had poor upright posture following the extended period of intubation, which may have contributed to the compression.

There are several potential complications that may occur after surgical removal of a hematoma (infection, bleeding, injury of adjacent structures); thus, we used a conservative treatment strategy to increase the space between the patient's clavicle and first rib to reduce the compression. After 2 weeks of treatment with a backpack bandage, the patient recovered completely.

Despite the fact that central venous catheterization is generally a low-risk procedure, mechanical complications occur in up to $18 \%$ of cases. ${ }^{1,2}$ In addition to failure to place the central venous catheter, arterial puncture, improper position, pneumothorax and hemothorax are common complications. Rare complications include plexus lesion, mediastinal hematoma or bleeding, and air embolism. Mechanical complications are associated with catheter insertions during the night, duration of catheter insertion and placement, number of needle passes and the patient's body mass index. ${ }^{2,3}$ Additional risk factors for a hematoma after catheter insertion include thrombopenia, oral anticoagulation, inherited bleeding disorders and catheter size.

\section{Roger W. Simon MD \\ Beatrice R. Amann-Vesti MD \\ Angiology Division \\ Department of Internal Medicine \\ University Hospital Zurich \\ Zurich, Switzerland}

This article has been peer reviewed.

Competing interests: None declared.

\section{REFERENCES}

I. Eisen LA, Narasimhan M, Berger JS, et al. Mechanical complications of central venous catheters. J Intensive Care Med 2006;2I:5I-3.

2. Merrer J, De Jonghe B, Golliot F, et al. Complications of femoral and subclavian venous catheterization in critically ill patients: a randomized controlled trial. JAMA 200I;286:700-7.

3. Takeyama $H$, Taniguchi $M$, Sawai $H$, et al. Limiting vein puncture to three needle passes in subclavian vein catheterization by the infraclavicular approach. Surg Today 2006;36:779-82. 